\title{
SOME FORMS OF RISK REGULATION IN SOLVENCY II
}

\section{Tomáš Cipra, Radek Hendrych*}

\begin{abstract}
The contribution deals with the risk regulation in the framework of Solvency II, which is the new regulatory system in insurance valid in majority of the EU countries since 2016. It concentrates on the underwriting risk (in particular, on the reserve risk) and on the counterparty default risk (i.e. mainly on the reinsurers' default risk), since such risks are crucial for insurance activities. Various actuarial approaches to the underwriting risk applied by subjects respected by insurance regulators and supervisors are surveyed. Moreover, one of them suggests by means of a real data example a simplified approach to the reserve risk, which may be appreciated in practice just for its simplicity. As to the counterparty default risk, the paper presents a method that can be suitable when the reinsurers form a small group of heterogeneous subjects imperilled by a common shock as a financial crisis or a natural catastrophe; this methodological approach is also demonstrated by a numerical example.
\end{abstract}

Keywords: risk regulation, Solvency II, underwriting risk, reserve risk, technical provisions, counterparty default risk, actuarial methods

JEL Classification: G22, G28, C02

\section{Introduction}

The preparatory work for Solvency II goes on since the beginning of the millennium. Solvency II has been the new regulatory system in insurance valid in majority of the EU countries since 2016. It is an innovative approach in the insurance regulation, which allows to quantify the corresponding capital requirements really according to underlying risks in particular insurance companies. It regulates mainly by means of solvency capital requirements all types of risks common in the modern insurance industry (underwriting risk, market risk, credit risk, liquidity risk, operational risk and others). Moreover, it copies the successful organization to three pillars from bank regulatory systems Basel II and III (see BIS, 2010).

The following points characterize the regulatory system Solvency II in a nutshell (see e.g. Cipra, 2015; Sandström, 2011):

* Tomáš Cipra, Department of Probability and Mathematical Statistics, Faculty of Mathematics and Physics, Charles University in Prague, Prague, Czech Republic (cipra@karlin.mff.cuni.cz); Radek Hendrych, Department of Probability and Mathematical Statistics, Faculty of Mathematics and Physics, Charles University in Prague, Prague, Czech Republic (hendrych@karlin.mff.cuni.cz). This work was supported by the Czech Science Foundation (the Grant No. GA P402/12/G097:

"DYME - Dynamic Models in Economics"). 
- $\quad$ Timing: The Solvency II regime has been valid in majority of EU countries since 2016. It includes transitional arrangements in a number of areas timed up until 2019.

- Jurisdiction: Solvency II is an obligatory regime for most insurers and reinsurers with their head office in the EU, including mutuals and companies in run-off unless their annual premium income is less than a prescribed sum depending on the national legislative. However, Solvency II should be applied in a way which is proportionate to the nature, scale and complexity of the insurer.

- Risk-based capital: Solvency II is a risk-based capital regime, similar in concept to Basel II, based on three pillars. Pillar 1 is a market consistent calculation of insurance liabilities and risk-based calculation of capital. Pillar 2 is a supervisory review process. Pillar 3 includes reporting and transparency requirements.

- Capital calculation:

- The calculation of insurance liabilities under Solvency II denoted as technical provisions includes a best estimate of liabilities and a risk margin (where the liability is not appropriately hedged).

- Capital is known as own funds and is divided into three Tiers (1-3) reflecting the ability to absorb losses. The risk based capital requirement, the solvency capital requirement $\mathrm{SCR}$, is calculated using either (i) a standard formula; or (ii) an internal model approved by the insurer's supervisor, or a mixture of both. The standard formula will cover the underwriting risk, market risk, credit risk and operational risk in a formulaic way. The calculation will be calibrated to ensure a 99.5 per cent confidence level over a one year period (the BBB rating according to Standard and Poor's should correspond to this risk level). Breach of the SCR results in supervisory intervention designed to restore the SCR level of capital.

- There is also the minimum capital requirement MCR set at a lower threshold (e.g. about 85 per cent confidence level). The MCR should not be less than 25 per cent of the SCR. Breach of the MCR (unless corrected quickly) leads to a loss of the insurer's authorisation.

- Assets: The rules requiring investment of assets in a list of admissible assets and the counterparty and asset limits contained in the previous regulatory regime Solvency I regime for EU insurers are replaced by the prudent person principle. This will place greater responsibility for investment decisions on the insurer including significantly increased reporting requirements in relation to assets.

- Groups: In the case of groups one can calculate the group SCR requirement based on a consolidated basis. This can reflect diversification but there may be problems, e.g. non-EU subsidiaries should calculate the solvency on a Solvency II basis with exceptions of some countries outside of the EU (e.g. Switzerland).

- $\quad$ Risk mitigation: Solvency II also includes rules relating to risk mitigation techniques and reinsurance. For non-EU based reinsurers they should have credit of equivalence according to their countries (e.g. EIOPA has assessed Bermuda, Switzerland and Japan as equivalent for the purposes of reinsurance). 
Figure 1 | Risks Covered by Solvency II Structured according to the Standard Formula (SLT denotes Similar to Life Techniques, i.e. risk components of health insurance similar from the technical point of view to life insurance, and Non-SLT in the opposite case; other symbols and terms are described in the text above)

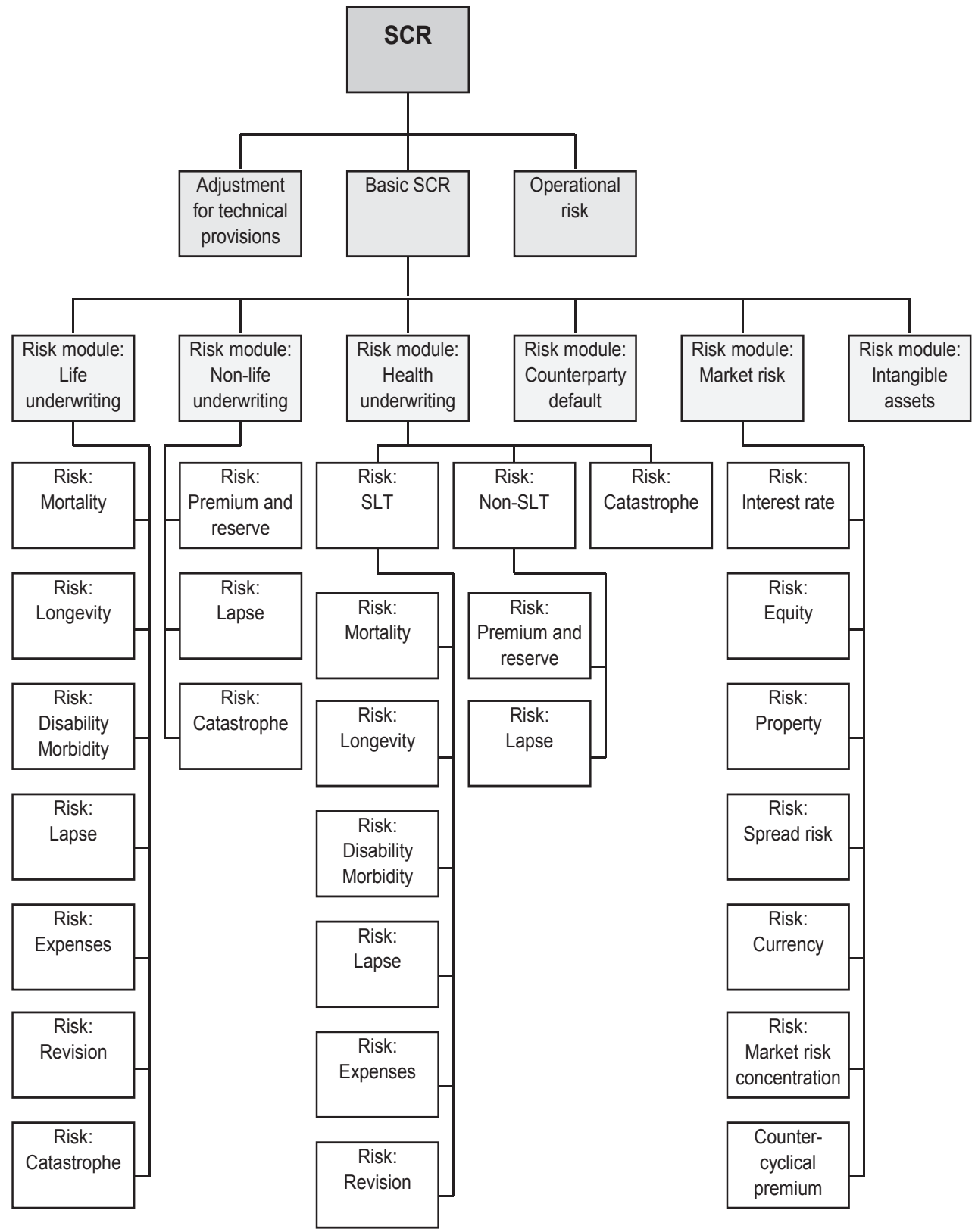


- Governance: Solvency II imposes formal governance requirements establishing roles such as a risk management function, an audit function, a compliance function and an actuarial function. In particular, the risk management should be set out in an Own Risk and Solvency Assessment (ORSA). The ORSA should include a risk-based assessment of the insurer's solvency needs based on its business and its own risk appetite (the supervisor will review it in the framework of the Pillar 2). Governance deals also with the outsourcing in the insurance company.

- Reporting: Pillar 3 requires an intensive reporting insurer's activity including a private regular supervisory report, a public annual report called the solvency and financial condition report (SFCR) and quantitative reporting templates (QRT).

- Supervision: The role of the insurance supervisor is very important and the success of Solvency II depends on its implementation by insurance supervisors in a consistent manner. It is intended that in group situations the relevant supervisors led by the group supervisor will cooperate and act in a coordinated manner.

To present the whole spectrum of risks taken into account by Solvency II one can use the survey in Figure 1 structuring the particular risks according to the standard formula.

This paper concentrates only on the underwriting risk (in particular, on the reserve risk) and on the counterparty default risk (i.e. mainly on the reinsurers' default risk), since such risks are crucial for insurance activities. Section 2 surveys from the actuarial point of view various approaches to the underwriting risk suggested by subjects respected by insurance regulators and supervisors; such a survey, not summarized in literature so far, can be useful for the European actuarial practice. Sections 3 and 4 concentrate on the reserve risk including the role of technical provisions in Solvency II. Particularly, in Section 4 one also suggests by means of a real data example a simplified approach to the reserve risk, which may be appreciated in practice just for its simplicity. Finally, Section 5 deals with the counterparty default risk and suggests a method that can be suitable when the counterparties (i.e. mainly reinsurers) form a small group of heterogeneous subjects impacted by a common shock as a financial crisis or a natural catastrophe (so far only "asymptotic" methods for large portfolios of homogenous risks are usual in literature); this method is also demonstrated by a numerical example.

\section{Various Approaches to Underwriting Risk}

The underwriting risk plays the key role in insurance industry. As the underwriting risk is concerned we usually distinguish premium risk and reserve risk. The premium risk consists of a possibility that the premium will not be sufficient to cover the insurer's obligations following from insurance contracts (mainly the claim expenditures). The reserve risk (or technical provision risk or run-off risk) is the risk of losses due to inadequate technical provisions and their spending. The approaches to underwriting risk described in this section usually result in calculation of the risk capital (or regulatory capital) RC, which is capable to mitigate losses caused by this risk (one can look upon it as a charge for the risk). Moreover, it is suitable to distinguish here the life and non-life insurance as it could be seen in the following examples. 


\subsection{GDV model in non-life insurance}

The final version of this model was published by GDV (Gesamtverband der Deutschen Versicherungswirtschaft) in 2005 as the German contribution to the discussion of the future form of the standard formula in Solvency II. However, as soon as the CEIOPS (i.e. the predecessor of EIOPA) published other suggestions, the development of the GDV model was stopped. In the context of underwriting risk of LoB $k$ (line of business), the GDV model uses the combined ratio

$$
C R_{k}=\frac{N C E_{k}+O E_{k}}{N P_{k}}
$$

where $N C E_{k}$ are the net claims expenditures (i.e. the run-off results for claims related to the insurance period plus the claims expenditures for the accounting year), $O E_{k}$ are the operating expenses and $N P_{k}$ are the net earned premiums for the given $\mathrm{LOB}_{k}$. For the combined ratio of the whole portfolio it could be written

$$
C R=\frac{\sum_{k}\left(N C E_{k}+O E_{k}\right)}{\sum_{k} N P_{k}}=\sum_{k} w_{k} C R_{k} \text { with } w_{k}=\frac{N P_{k}}{\sum_{k} N P_{k}},
$$

where $C R$ denotes the combined ratio of the whole insurance portfolio and $C R_{k}$ denotes the combined ratio of the $k$-th LOB (we use symbols from GDV (2005)). If the particular combined ratios are looked upon as random variables with expectations $\mu_{k}=\mathrm{E}\left(C R_{k}\right)$ and variances $\sigma_{k}^{2}=\operatorname{var}\left(C R_{k}\right)$, then it holds for $\mu=\mathrm{E}(C R)$ and $\sigma^{2}=\operatorname{var}(C R)$

$$
\mu=\sum_{k} w_{k} \mu_{k}, \quad \sigma^{2}=\sum_{j} \sum_{k} w_{j} w_{k} \sigma_{j} \sigma_{k} \rho_{j k},
$$

where $\rho_{j k}$ is the correlation coefficient between $C R_{j}$ and $C R_{k}$. Let $S R=R C / N P$ be the corresponding solvency ratio. The principle of GDV model consists in the requirement that the loss of each insurer $N C E+O E-N P$ should not exceeds the risk capital $R C$ with a high probability $\alpha$ (e.g. $99.5 \%$ ), i.e. $P(N C E+O E-N P \leq R C) \geq \alpha$, i.e. $\mathrm{P}(C R-1 \leq S R) \geq \alpha$. It can be rewritten by means of the $\alpha$-quantile $q$ of CR as

$$
S R \geq q_{\alpha}-1
$$

In practice one can approximate this quantile as $q_{\alpha} \approx \mu+a \cdot \sigma$, where the coefficient $a$ would be prescribed by the regulator applying global market data. Therefore the required risk capital must be at least

$$
R C \approx N P \cdot(\hat{\mu}+a \cdot \hat{\sigma}-1)
$$

where $\hat{\mu}$ and $\hat{\sigma}$ are estimates of $\mu$ and $\sigma$ delivered individually by particular insurers over their LOBs using the estimated portfolio relations (3). 


\subsection{CEA model in non- life insurance}

Similarly as in the previous case, the CEA model (Comite Europeen des Assurances) was suggested during the discussion of the standard formula in Solvency II (see CEA, 2006). All calculations are again made for separate LOBs and aggregated using correlations:

The reserve risk in $\mathrm{LOB}_{k}$ requires the risk capital $R C_{R R, k}$ calculated as

$$
R C_{R R, k}=f_{R R, k} \cdot C P_{k},
$$

where $C P_{k}$ are net claims provisions and $f_{R R, k}$ is a reserve risk factor prescribed as a standard for all insurers (similarly as in the GDV model $f_{R R, k}$ is taken as a multiple of the standard deviation of the distribution of the technical provisions such that the market value of technical provisions with the corresponding risk capital are $99.5 \%$ sufficient to cover the incurred claims in the run-off sense).

The premium risk in $\mathrm{LOB}_{k}$ requires the risk capital $R C_{P R, k}$ calculated as

$$
R C_{P R, k}=f_{P R, k} \cdot\left(W P_{k}+U P_{k}\right),
$$

where $W P_{k}$ is the net written premium, $U P_{k}$ is the net unearned premium and $f_{P R, k}$ is a premium risk factor prescribed as a standard for all insurers (again it is a multiple of the standard deviation of the distribution of the loss ratio such that the net premium written in the coming year plus the net unearned premium reserve altogether with the corresponding risk capital are $99.5 \%$ sufficient to cover all insurer's expenditures (i.e. the claims, the claim reserves and operating expenses).

\subsection{IAA model in life insurance}

Though the International Actuarial Association deals with all types risk (see e.g. IAA, 2004) we shall present here only its contribution to mortality risk as an important biometrical component of the underwriting risk in the framework of life insurance. In any case, the mortality risk plays a key role for underwriting activities of life companies and its risk components are volatility, catastrophes, level uncertainty and trend uncertainty. The IAA model supposes the common situation that the portfolio of a given life insurer is a set of individual contracts with death probabilities $q_{i}$ and claims $X_{i}$ in the case of death $(i=1, \ldots, n)$. The aggregate claim is modelled as a random variable $S$ with the compound Poisson distribution and the first three moments

$$
\mu=\sum_{i=1}^{n} q_{i} X_{i}, \quad \sigma=\sqrt{\sum_{i=1}^{n} q_{i} X_{i}^{2}}, \quad \gamma=\frac{\sum_{i=1}^{n} q_{i} X_{i}^{3}}{\sigma^{3}} .
$$

Due to the equivalence principle, the premium $P$ should fulfill $P=\mu$. Then there can be calculated the risk capital for particular components of the mortality risk in the following way:

(i) Volatility: The term volatility means in the context of IAA model in life insurance the risk that the aggregate loss in the given portfolio fluctuates around the expected 
value $\mu$ and the premium may not cover the aggregate claim $S$. The IAA model suggests the application of VaR principle so that the risk capital $R C_{v o l}$ fulfills with a high confidence $\alpha$

$$
P\left(S \leq R C_{v o l}+P\right)=\alpha .
$$

From (9) one can express the risk capital $R C_{v o l}$ by means of the approximation denoted as NP2 (Normal Power 2, sometimes called also Cornish-Fisher extension) as

$$
R C_{v o l}=\sigma \cdot\left(\Phi^{-1}(\alpha)+\frac{\left(\Phi^{-1}(\alpha)\right)^{2}-1}{6} \gamma\right),
$$

where $\Phi^{-1}(\alpha)$ is the $\alpha$-quantile of $\mathrm{N}(0,1)$. Moreover, the IAA (2004) recommended the confidence level $99.5 \%$ so that

$$
R C_{v o l}=\sigma \cdot(2.58+0.94 \gamma) .
$$

Applying the simplifying approximation with an average mortality $q=2.5 \%$ one obtains even

$$
R C_{v o l}=\left(\frac{77.4}{\sqrt{n}}+\frac{942.7}{n}\right) \cdot P,
$$

where $n$ is the number of contracts in the portfolio (see Table 1 for three various portfolios with different $n$ and skewness of claims). A less sophisticated approximation which makes use of assumptions $q_{i} \sim q$ and $X_{i} \sim X$ leads to the formula

$$
R C_{v o l}=\left(\frac{\Phi^{-1}(\alpha)}{\sqrt{q n}}+\frac{\left(\Phi^{-1}(\alpha)\right)^{2}-1}{6 q n}\right) \cdot P .
$$

Table 1 | The Risk Capital Covering Volatility Component of Mortality Risk with Confidence Level $\mathbf{9 9 . 5} \%$ for Three Various Portfolios

\begin{tabular}{|l|c|c|c|}
\hline Portfolio & $\mathbf{n}$ & Skewness of claims & $\mathbf{R C}_{\text {vol }}(\%$ of $\mathbf{P})$ \\
\hline $\mathbf{1}$ & 125,970 & 0.13 & 22.8 \\
\hline $\mathbf{2}$ & 60,777 & 0.77 & 68.1 \\
\hline $\mathbf{3}$ & 24,570 & 0.38 & 57.4 \\
\hline
\end{tabular}

Source: IAA (2004)

(ii) Catastrophe: Since it is hard to model the catastrophe component of the mortality risk mathematically the IAA suggested that the corresponding risk capital could be based on the fixed ratio of the expected number of death in the given portfolio.

(iii) Level uncertainty: This uncertainty of the mortality risks consists in an inadequate estimate of the average mortality. According to the study of IAA the Table 2 presents the increase of the single premium caused by the shock increase of mortality by 
$10 \%$. Besides this scenario approach the risk capital covering the level uncertainty in the framework of the mortality risk can be calculated by means of the formula (12) with the confidence $99.5 \%$ : if e.g. the average mortality was estimated by means of a sample of 300000 yearly observations then the corresponding mortality shock should be $77.4 / \sqrt{ } 300000+942.7 / 300000=0.14=14 \%$ of the applied reference mortality.

Table 2 | The Increase of the Single Premium Caused by the Shock Increase of Mortality by $10 \%$

\begin{tabular}{|l|c|c|c|}
\hline \multirow{2}{*}{$\begin{array}{l}\text { Term of } \\
\text { contract }\end{array}$} & \multicolumn{3}{|c|}{ The increase of the single premium caused by the shock increase of mortality by $\mathbf{1 0} \%$} \\
\cline { 2 - 4 } & Endowment insurance & Pure endowment insurance & Term insurance \\
\hline $\mathbf{5}$ & $0.07 \%$ & $0.73 \%$ & $9.70 \%$ \\
\hline $\mathbf{1 0}$ & $0.19 \%$ & $1.14 \%$ & $9.49 \%$ \\
\hline $\mathbf{2 0}$ & $0.44 \%$ & $1.54 \%$ & $9.33 \%$ \\
\hline
\end{tabular}

Source: IAA (2004)

(iv) Trend uncertainty: This uncertainty of the mortality risks consists in an inadequate estimate of the mortality trend. The IAA (2004) suggested a simplified factor approach where the corresponding risk capital $R C_{\text {trend }}$ can be obtained if multiplying the single premium by the factor $\min (\alpha, n \cdot \beta)$ (see Table 3 for the coefficients $\alpha$ and $\beta$ ). In the case of life annuities one suggested to take $4 \%$ of the capitalized annuity value instead of this factor.

Table 3 | The Coefficients $\alpha$ and $\beta$ for the Calculation of the Risk Capital Covering Trend Uncertainty Component of Mortality Risk

\begin{tabular}{|l|c|c|}
\hline & $\boldsymbol{\alpha}(\%)$ & $\boldsymbol{\beta}(\%)$ \\
\hline Endowment insurance & 3 & 0.15 \\
\hline Pure endowment insurance & 7 & 0.35 \\
\hline Term insurance & 30 & 1.50 \\
\hline
\end{tabular}

Source: IAA (2004)

\section{Technical Provisions in Solvency II}

Solvency II promotes a different approach to the technical provisions in comparison with the previous practice. Also the regulations conform to this new practice (e.g. one discusses no upper limit of the technical interest rate in the life insurance, no investment limits for assets covering the technical provisions and other changes). It is motivated by the effort to construct the technical provisions in a market consistent way composing them from their best estimate and the risk margin (only in specific situations the technical provisions can be constructed as a whole by the mark-to-market method, e.g. when they can be reliably replicated or they are hedged by a suitable financial portfolio). 
The technical provisions are constructed to cover the future obligations of insurers. In comparison with the previous practice, Solvency II distinguishes only three types of technical provisions, namely

- $\quad$ for obligations from life insurance (the so called life insurance obligations);

- $\quad$ for premium in non-life insurance (the so called premium provision);

- $\quad$ for claims in non-life insurance (the so called provision for claims outstanding).

If an obligation cannot be replicated by means of suitable financial instruments, then the technical provisions $T P$ must by constructed as the best estimate $B E$ of this obligation discounted applying the risk-free interest rate and augmented by the risk margin $R M$ :

$$
T P=\text { discounted } B E+R M
$$

\subsection{Best estimate}

The best estimate $B E$ is the probability-weighted average (i.e. the expected or mean value) of future cash flows generated by the given obligation and covered by the technical provisions. When calculating $B E$, such actuarial or statistical methods should be used that reflect all risks affecting the corresponding cash flows. The calculation methods for $B E$ can be classified in the following way:

- Simulation methods consist in generating future scenarios, which are representative in an appropriate way for all future scenario alternatives. The recommended simulation procedures are e.g.:

- Simulation Monte Carlo: one simulates TP applying realizations of suitable random variables, and the mean of obtained values is regarded as the expected value of TP; in some cases, e.g. when simulating the lapses, the correlations with the development of relevant macroeconomic variables can be used.

- Bootstrap method: this method is popular in the actuarial context and is based on the resampling of estimated residuals in the corresponding actuarial model, which enables in the context of the technical provisions to obtain information on their distribution (see e.g. Hendrych and Cipra (2015) for life insurance).

- Bayes approach: when simulating TP one combines explicit assumptions on their prior distribution with the observed insurance claims.

- Analytical methods are based on explicit formulas for probability distribution of future cash flows. Examples can be given both for the life insurance (e.g. stochastic models of mortality) and for the non-life insurance (e.g. the distribution free Chain-Ladder models, see Section 4).

- Deterministic methods produce the projections of cash flows necessary for the calculation of $B E$ by means of deterministic procedures, the stochastic aspects being contained in the appropriate assumptions (e.g. Chain-Ladder, Cape Cod, Bornhuetter-Ferguson, separation method, see Cipra, 2015). 


\subsection{Discounting}

The discounting produces the present value of $B E$ using suitable risk-free interest rates. According to the Directive 2014/51/EU (the so called Omnibus II), EIOPA in a position of the central insurance subject in Europe will publish regularly for this purpose the so called basic risk-free interest rate, which is supposed to be modified in each domestic insurance market by means of the so called matching and volatility adjustment respecting the market creditibility and other factors.

\subsection{Risk margin}

Risk margin RM must guarantee that the technical provisions truly cover the corresponding obligations. $R M$ is usually calculated as the present value of capital costs necessary for such a guarantee (also here one applies the risk-free interest rate to discount these capital costs):

$$
R M=C o C \cdot \sum_{t \geq 0} \frac{S C R_{t}}{\left(1+r_{t+1}\right)^{t+1}},
$$

where $C o C$ is the cost capital rate (Cost-of-Capital) with a suggested starting value $6 \%$; $S C R_{t}$ is the solvency capital requirement after $t$ years to cover the obligations in technical provisions and $r_{t+1}$ is the risk-free interest rate for the maturity $t+1$ (see above).

Since the procedure according to (15) is too general, and there may be problems with its practical application, one admits some simplifications for the calculation of $R M$. The highest possible simplification consists in the naïve formula

$$
R M=\alpha_{L O B} \cdot B E_{0}^{\text {net }},
$$

where $B E_{0}{ }^{n e t}$ is the best estimate of the net $T P$ calculated in time $t=0$, i.e. excluding reinsurance, and $\alpha_{L O B}$ is a fixed ratio for the given LOB. The more sophisticated formulas for the risk margin are created individually for particular risk modules and submodules (e.g. separately for underwriting, market and credit risk).

Moreover, the construction of technical provisions according to Solvency II respects some general principles:

(i) Segmentation: When calculating the technical provisions the insurers segment their insurance obligations into homogeneous risk groups. Moreover, the assignment of an obligation to a LOB should reflect the nature (substance) of the risks relating to the given obligation, which may not reflect the legal form: e.g. obligations for health insurance should be allocated to life or non-life according to whether the business is carried out on a similar technical basis to that of life insurance (SLT health) or to that of non-life insurance (non-SLT health, see also Figure 1). The process of segmentation of insurance contracts into particular risks is called unbundling (e.g. contracts covering life insurance can be unbundled to SLT health insurance, life insurance with profit participation, index-linked life insurance, unit-linked life insurance and other life insurance). 
(ii) Contract boundary: There are various obligations relating to a given insurance contract, and the principle of contract boundary determines which of them really belong into the contract framework ("boundaries").

(iii) Recoverables from reinsurance contracts: The best estimate of technical provisions is calculated in the gross form, i.e. without subtracting sums recoverable from reinsurance contracts. However, the results concerning the recoverables must be adjusted to take account of expected losses due to the default of counterparty (i.e. due to the default of reinsurer). That adjustment is based on an assessment of the probability of default $P D$ and on the average loss resulting there from (the so called loss-given-default $L G D$ ). For instance, let the recoverables in the first, second and third year amount to $R_{1}, R_{2}$ and $R_{3}$, respectively, with the same $L G D$ of $70 \%$, i.e. the recovery amounts to $30 \%$ of the loss. Then the recoverables should be reduced by the amount

$$
0.7 \cdot\left[P D_{1} \cdot\left(R_{1}+R_{2}+R_{3}\right)+P D_{2} \cdot\left(R_{2}+R_{3}\right)+P D_{3} \cdot R_{3}\right]
$$

(for simplicity we ignore the time value of money).

(iv) Proporcionality of TP method: Insurers apply for the calculation of technical provisions such methods that are proportionate to nature, scale and complexity of risks related to the given insurance obligations. Judging the proportionality of a method for $T P$ calculation the insurer should assess the administrative and calculation burden of the method (both in a quantitative and qualitative way) respecting the complexity of all risks affecting the technical provisions. In particular, according to this principle the insurer is in some cases allowed to use suitable simplifications (see Section 4).

When calculating TP one must also regard a lot of further aspects, e.g. all overhead expenses and costs related to the management of insurance and reinsurance obligations, the (cost) inflation, future profit shares (even if they are not guaranteed) and others.

\section{Calculation of Risk Capital for Covering the Reserve Risk}

In this section we present three approaches to the calculation of risk capital $R C$ to cover the reserve risk. These approaches have been chosen since all of them are applicable in the framework of Solvency II (e.g. as the internal model), but they were unusual for the technical provisions in the periods before Solvency II (we ignore a lot of other methods, e.g. Hendrych and Cipra, 2015; Merz and Wüthrich, 2008; SST, 2006).

\subsection{One-year time horizon approach}

The classical claim reserving methods for the non-life insurance have an ultimo time horizon, i.e. they look at the total run-off. In spite of this fact, the one-year time horizon reserving methods have been suggested supported by the practical opinion that they are sufficient for the regulatory purposes of solvency (see e.g. Hürlimann, 2008; and Ohlsson and Lauzeningks, 2008): 
The reserve risk in such a one-year time horizon model is taken into account by means of the ratio $L R$ (reserve loss ratio) that expresses the considered loss in relation to the claim reserve:

$$
L R_{R}=\frac{L}{R^{0}}=\frac{R^{01}+C^{01}-R^{0}}{R^{0}}=\frac{R^{01}+C^{01}}{R^{0}}-1=X-1,
$$

where $R^{0}$ is the claim reserve in the beginning of the current year (i.e. in the beginning of the considered one-year period), $R^{01}$ is the best estimate of this reserve at the end of the current year (the upper index indicates that $R^{01}$ includes the changes occurred in the reserve during this one-year period) and $C^{01}$ is the estimate of the amount paid out during the current year. Obviously the ratio $L R$ relates the technical loss $L$ (the run-off result) to the reserve $R^{0}$ in the beginning of the current year.

Due to the actuarial principle of equivalence it must hold $\mathrm{E}(L R)=0$, or equivalently $\mathrm{E}(X)=1$. Empirical data show (e.g. Hürlimann, 2008) that the random variable $X$ can be modelled by means of the logarithmic normal distribution

$$
X \sim L N\left(\mu_{X}, \sigma_{X}^{2}\right)
$$

Since then $\mathrm{E}(X)=\exp \left(\mu_{X}+\sigma_{X}^{2} / 2\right)=1$ and $\operatorname{var}(X)=\exp \left(2 \mu_{X}+\sigma_{X}^{2}\right)\left(\exp \sigma_{X}^{2}-1\right)=\sigma^{2}$, it must hold $\mu_{X}=-\sigma_{X}^{2} / 2$ and $\sigma_{X}^{2}=\ln \left(1+\sigma^{2}\right)$.

By means of these results we can find an analytical formula for the value-at-risk $\operatorname{VaR}_{\alpha}(L)$ of the loss $L$ in the numerator of the ratio $L R$, i.e. the analytical formula for the risk capital required with a given confidence to cover the reserve risk in the model with one-year time horizon (see also Cipra, 2015):

$$
\begin{aligned}
& R C=\operatorname{VaR}_{\alpha}(L)=\left(\operatorname{VaR}_{\alpha}\left(L / R^{0}\right)\right) \cdot R^{0}=\left(\operatorname{VaR}_{\alpha}(X-1)\right) \cdot R^{0}=\left(\operatorname{VaR}_{\alpha}(X)-1\right) \cdot R^{0}= \\
& =\left(\exp \left\{\mu_{X}+\sigma_{X} \Phi^{-1}(\alpha)\right\}-1\right) \cdot R^{0}=\left(\frac{\exp \left\{\Phi^{-1}(\alpha) \cdot \sqrt{\ln \left(1+\sigma^{2}\right)}\right\}}{\sqrt{1+\sigma^{2}}}-1\right) \cdot R^{0} .
\end{aligned}
$$

Quite analogously we can derive the reserve risk capital $R C$ when one applies the expected shortfall $E S_{\alpha}(L)$ as the risk measure (Cipra, 2015):

$$
R C=E S_{\alpha}(L)=\frac{\alpha-\Phi\left(\Phi^{-1}(\alpha) \cdot \sqrt{\ln \left(1+\sigma^{2}\right)}\right)}{1-\alpha} \cdot R^{0}
$$

Table 4 presents the risk capital for the confidence levels $99 \%$ and $99.5 \%$ in the one-year time horizon depending on the standard deviation $\sigma$ of the ratio $X$ and on the claim reserve $R^{0}$ in the beginning of the current year. E.g. the reserve risk capital for the estimated standard deviation 0.12 of the ratio $X$ calculated as the expected shortfall $E S_{0.99}$ is

$$
R C=E S_{0.99}=3.054 \cdot 0.12 \cdot R^{0}
$$

(the standard deviation must be estimated using the historical observations of $X$ ). 
Table 4 | Reserve Risk Capital for the Confidence Levels $99 \%$ and $99.5 \%$ in the One-Year Time Horizon Depending on the Standard Deviation $\sigma$ of the Ratio $X$ and on the Claim Reserve $\mathbf{R}^{0}$ in the Beginning of the Current Year

\begin{tabular}{|l|c|c|c|c|}
\hline & \multicolumn{2}{|c|}{$V a \boldsymbol{R}_{\boldsymbol{a}}$} & \multicolumn{2}{c|}{$\boldsymbol{S S}_{\boldsymbol{a}}$} \\
\hline $\boldsymbol{\sigma}$ & $\boldsymbol{a}=\mathbf{0 . 9 9}$ & $\boldsymbol{a}=\mathbf{0 . 9 9 5}$ & $\boldsymbol{a}=\mathbf{0 . 9 9}$ & $\boldsymbol{a}=\mathbf{0 . 9 9 5}$ \\
\hline $\mathbf{0 . 1 2}$ & $2.594 \cdot \sigma \cdot R^{0}$ & $2.925 \cdot \sigma \cdot R^{0}$ & $3.054 \cdot \sigma \cdot R^{0}$ & $3.366 \cdot \sigma \cdot R^{0}$ \\
\hline $\mathbf{0 . 1 3}$ & $2.617 \cdot \sigma \cdot R^{0}$ & $2.955 \cdot \sigma \cdot R^{0}$ & $3.088 \cdot \sigma \cdot R^{0}$ & $3.408 \cdot \sigma \cdot R^{0}$ \\
\hline $\mathbf{0 . 1 4}$ & $2.639 \cdot \sigma \cdot R^{0}$ & $2.985 \cdot \sigma \cdot R^{0}$ & $3.122 \cdot \sigma \cdot R^{0}$ & $3.450 \cdot \sigma \cdot R^{0}$ \\
\hline $\mathbf{0 . 1 5}$ & $2.661 \cdot \sigma \cdot R^{0}$ & $3.015 \cdot \sigma \cdot R^{0}$ & $3.156 \cdot \sigma \cdot R^{0}$ & $3.492 \cdot \sigma \cdot R^{0}$ \\
\hline $\mathbf{0 . 1 6}$ & $2.684 \cdot \sigma \cdot R^{0}$ & $3.045 \cdot \sigma \cdot R^{0}$ & $3.190 \cdot \sigma \cdot R^{0}$ & $3.535 \cdot \sigma \cdot R^{0}$ \\
\hline $\mathbf{0 . 1 7}$ & $2.706 \cdot \sigma \cdot R^{0}$ & $3.075 \cdot \sigma \cdot R^{0}$ & $3.224 \cdot \sigma \cdot R^{0}$ & $3.578 \cdot \sigma \cdot R^{0}$ \\
\hline
\end{tabular}

Source: authors

\subsection{Analytical approach to technical provisions}

Analytical methods are based on explicit formulas for probability distribution of future cash flows. For instance, Mack (1993) has derived the formula for the mean squared error MSE of the claim reserve constructed by the method Chain-Ladder (e.g. Cipra, 2015), which is important for description of the stochastic behaviour of this reserve both of the type RBNS (i.e. Reported But Not Settled) and the type IBNR (i.e. Incurred But Not Reported). In any case, it is a typical example of the analytical approaches to technical provisions in practice:

$$
\operatorname{MSE}\left(\hat{R}_{i}\right)=\hat{C}_{i I}^{2} \cdot \sum_{k=I+1-i}^{I-1} \frac{\hat{\sigma}_{k}^{2}}{\hat{f}_{k}^{2}}\left(\frac{1}{\hat{C}_{i k}^{2}}+\frac{1}{\sum_{j=1}^{I-k} C_{j k}}\right),
$$

where

$C_{i k}$ is a random variable denoting the cumulative claim amount paid out till the development year $k$ since the accident year $i(i, k=1, \ldots, I)$; one ignores the dependencies among various accident years so that the random vectors $C_{i 1}, \ldots \ldots, C_{i I}$ and $C_{j 1}, \ldots, C_{j I}$ are supposed to be independent for $i \neq j$;

$R_{i} \quad$ is the claim reserve for the accident year $i$, i.e. $R_{i}=C_{i I}-C_{i, I+1-i}(i=2, \ldots, I)$;

$f_{k} \quad$ is the development coefficient in $\mathrm{E}\left(C_{i, k+1} \mid C_{i 1}, \ldots, C_{i k}\right)=C_{i, k} \mid f_{k}(i=1, \ldots, I, k=1, \ldots$, $I-1)$ which can be estimated in the unbiased way by the method of Chain-Ladder (e.g. Cipra, 2010) as

$$
\hat{f}_{k}=\sum_{j=1}^{I-k} C_{j, k+1} / \sum_{j=1}^{I-k} C_{j, k}, k=1, \ldots, I-1
$$


(moreover, these estimates of development coefficients are mutually uncorrelated); then in the Chain-Ladder method one estimates future cumulative claim amounts as

$$
\hat{C}_{i k}=C_{i, I+1-i} \cdot \hat{f}_{I+1-i} \cdot \ldots \cdot \hat{f}_{k-i}, k>I+1-i \text { and } \hat{C}_{i, I+1-i}=C_{i, I+1-i} ;
$$

$\sigma_{k}$ is the unknown parameter in $\operatorname{var}\left(C_{i, k+1} \mid C_{i 1}, \ldots, C_{i k}\right)=C_{i k} \mid \sigma_{k}^{2}(i=1, \ldots, I, k=1, \ldots, I-1)$ which can be estimated in the unbiased way as

$$
\hat{\sigma}_{k}^{2}=\frac{1}{I-k-1} \sum_{i=1}^{I-k} C_{i k}\left(\frac{C_{i, k+1}}{C_{i k}}-\hat{f}_{k}\right)^{2}, k=1, \ldots, I-2 ;
$$

$\operatorname{MSE}\left(\hat{R}_{i}\right)$ is the estimated mean squared error of the claim reserve for the claim amounts with the accident year $i$ defined as $\mathrm{E}\left(\hat{R}_{i}-R_{i} \mid C_{i 1}, \ldots, C_{I+1-i}\right)^{2}$.

Since the insurer needs the amount of total reserve $R=R_{2}+\ldots+R_{I}$, one must extend the formula (22) to the form

$$
\operatorname{MSE}(\hat{R})=\sum_{i=2}^{I}\left(\operatorname{MSE}\left(\hat{R}_{i}\right)+\hat{C}_{i I} \cdot \sum_{j=i+1}^{I} \hat{C}_{j I} \cdot \sum_{k=I+1-i}^{I-1} \frac{2 \hat{\sigma}_{k}^{2} / \hat{f}_{k}}{\sum_{n=1}^{I-k} C_{n k}}\right) .
$$

\section{Table 5 | Cumulative Run-Off Triangle}

\begin{tabular}{|l|c|c|c|c|c|c|c|c|c|}
\hline \multirow{2}{*}{$\begin{array}{l}\text { Accident } \\
\text { year }\end{array}$} & \multicolumn{7}{|c|}{ Development year } \\
\cline { 2 - 11 } & $\mathbf{1}$ & $\mathbf{2}$ & $\mathbf{3}$ & $\mathbf{4}$ & $\mathbf{5}$ & $\mathbf{6}$ & $\mathbf{7}$ & $\mathbf{8}$ & $\mathbf{9}$ \\
\hline $\mathbf{1}$ & 58,046 & 127,970 & 467,599 & $1,027,692$ & $1,360,489$ & $1,647,310$ & $1,819,179$ & $1,906,852$ & $1,950,105$ \\
\hline $\mathbf{2}$ & 24,492 & 141,767 & 984,288 & $2,142,656$ & $2,961,978$ & $3,683,940$ & $4,048,898$ & $4,115,760$ & \\
\hline $\mathbf{3}$ & 32,848 & 274,682 & $1,522,637$ & $3,203,427$ & $4,445,927$ & $5,158,781$ & $5,342,585$ & & \\
\hline $\mathbf{4}$ & 21,439 & 529,828 & $2,900,301$ & $4,999,019$ & $6,460,112$ & $6,853,904$ & & & \\
\hline $\mathbf{5}$ & 40,397 & 763,394 & $2,920,745$ & $4,989,572$ & $5,648,563$ & & & & \\
\hline $\mathbf{6}$ & 90,748 & 951,994 & $4,210,640$ & $5,866,482$ & & & & & \\
\hline $\mathbf{7}$ & 62,096 & 868,480 & $1,954,797$ & & & & & & \\
\hline $\mathbf{8}$ & 24,983 & 284,441 & & & & & & & \\
\hline $\mathbf{9}$ & 13,121 & & & & & & & & \\
\hline
\end{tabular}

Source: Mack (1993)

For instance, let us consider the run-off triangle in Table 5 (for the building insurance in the framework of mortgage portfolio) applied for the IBNR reserve creation. The estimated reserves for particular accident years including the total reserve estimated over all development years by the Chain-Ladder method are given in Table 6. Moreover, 
the corresponding standard deviations (i.e. root mean squared errors $\sqrt{ } \operatorname{MSE}\left(\hat{R}_{i}\right)$ Rand $\sqrt{ } \operatorname{MSE}(\hat{R})$ presented as the percentage of the estimated reserves) are calculated in Table 6 according to the formulas (22) and (26). One can see that the errors of the Chain-Ladder reserves can be unexpectedly high so that the corresponding risk capital $R C$ must take this fact into account in the proper way (see also Wüthrich and Merz, 2008).

Table 6 | The IBNR Reserves Estimated by the Mack's Method Including Their Standard Deviations (i.e. the root mean squared errors presented as the percentage of the estimated reserves)

\begin{tabular}{|l|c|c|}
\hline $\boldsymbol{i}$ & $\hat{R}_{i}$ & $\begin{array}{c}\text { Standard } \\
\text { deviation (\%) }\end{array}$ \\
\hline $\mathbf{2}$ & 93 & 65 \\
\hline $\mathbf{3}$ & 265 & 53 \\
\hline $\mathbf{4}$ & 834 & 38 \\
\hline $\mathbf{5}$ & 1,568 & 38 \\
\hline $\mathbf{6}$ & 3,696 & 28 \\
\hline $\mathbf{7}$ & 3,487 & 37 \\
\hline $\mathbf{8}$ & 2,956 & 61 \\
\hline $\mathbf{9}$ & 1,647 & 133 \\
\hline Total & 14,547 & 26 \\
\hline
\end{tabular}

Source: Mack (1993)

\subsection{Some simplifications}

Reasonable simplifications for the TP calculations are acceptable in some cases in the framework of Solvency II. For instance, one can calculate the IBNR reserve simply as

$$
T P_{I B N R}=f_{L O B} \cdot T P_{R B N S},
$$

where $f_{L O B}$ is a factor specific for the given LOB of the given insurer. This factor expresses the approximate ratio of the IBNR reserve from the RBNS reserve where the RBNS reserve is composed by particular case estimates provided by experts.

Here we suggest a more sophisticated approach using observations over several years (in particular over three years $t, t-1$ and $t-2$ ). The corresponding formula has the form

$$
T P_{I B N R, t}=N_{t} \cdot A_{t},
$$

where

$$
N_{t}=R_{t} \cdot k_{t}=R_{t} \cdot \frac{N_{t-1} / p_{1}+N_{t-2} / p_{2}+N_{t-3}}{R_{t-1}+R_{t-2}+R_{t-3}}
$$


$A_{t} \quad$ is the mean claim amount of the type IBNR in year $t$;

$N_{t-i}$ is the number of claims of the type IBNR in year $t-i$ independently on their accident year (this value is observed for $i=1,2,3$ and $N_{t}$ must be calculated additionally according to (29));

$p_{1} \quad$ is the percentage of claims of the type IBNR in the end of year $t-3$ reported during year $t-2$;

$p_{2}$ is the percentage of claims of the type IBNR in the end of year $t-3$ reported during years $t-2$ and $t-1$;

$R_{t-i}$ is the number of claims reported in year $t-i$ independently on their accident year (this value is observed for $i=0,1,2,3$ ).

When calculating the rate coefficient $k_{t}$ in (29) one must regard the fact that e.g. the observed number of $N_{t-1}$ in year $t-1$ is incomplete and one should augment it artificially to the actual (but unobserved) number of claims of this type by means of the observed percentage $p_{1}$. Moreover, the proper calculation is more sophisticated, since it should include the estimation bias, the inflation, the time value of money and other relevant facts (see the following numerical example).

Let us demonstrate the second approach to simplified IBNR reserves by means of the data till 12 December 2015 and additional information from Tables 7 and 8 . Using this information one constructs the best estimate of IBNR reserve to this date.

Table 7 | Simplified Calculation of the IBNR Reserve on 31 December 2015: Data and Projections

\begin{tabular}{|l|c|c|c|c|}
\hline $\boldsymbol{t}$ & $\begin{array}{c}\boldsymbol{N}_{\boldsymbol{t}} \\
\text { (pcs) }\end{array}$ & $\begin{array}{c}\boldsymbol{R}_{\boldsymbol{t}} \\
\text { (pcs) }\end{array}$ & $\begin{array}{c}\text { Estimated inflation } \\
(\%)\end{array}$ & $\begin{array}{c}\text { Estimated discount rate } \\
\text { (\%) }\end{array}$ \\
\hline $\mathbf{2 0 1 2}$ & 100 & 8,700 & - & - \\
\hline $\mathbf{2 0 1 3}$ & 100 & 8,200 & - & - \\
\hline $\mathbf{2 0 1 4}$ & 90 & 8,500 & - & - \\
\hline $\mathbf{2 0 1 5}$ & - & 10,500 & - & 4 \\
\hline $\mathbf{2 0 1 6}$ & - & - & 5 & 4 \\
\hline $\mathbf{2 0 1 7}$ & - & - & 5 & 4 \\
\hline $\mathbf{2 0 1 8}$ & - & - & 5 & 4 \\
\hline
\end{tabular}

Source: authors

The claims reported to 31 December 2015 amount to 11,000,000 EUR. For one half of this sum (i.e. for 5,500,000 EUR) one has created the RBNS reserve requiring the risk margin due to a possible bias of the estimate to $90 \%$ of actual value so that the original amount of $11,000,000$ EUR is corrected to 5,500,000 $/ 0.9+5,500,000=11,611,111 \mathrm{EUR}$ (the so called bias correction). 


\begin{tabular}{|l|c|c|c|c|}
\hline $\boldsymbol{t}$ & $\begin{array}{c}\text { Delay in claims } \\
\text { (\%) }\end{array}$ & $\begin{array}{c}\text { Delay in claims } \\
\text { (EUR) }\end{array}$ & $\begin{array}{c}\text { Inflation } \\
\text { (EUR) }\end{array}$ & $\begin{array}{c}\text { Discounting } \\
\text { (EUR) }\end{array}$ \\
\hline $\mathbf{2 0 1 5}$ & 50 & $5,805,556$ & $6,095,833$ & $5,861,378$ \\
\hline $\mathbf{2 0 1 6}$ & 35 & $4,063,889$ & $4,480,438$ & $4,142,416$ \\
\hline $\mathbf{2 0 1 7}$ & 15 & $1,741,667$ & $2,016,197$ & $1,792,392$ \\
\hline Total & - & $11,611,111$ & - & $11,796,186$ \\
\hline
\end{tabular}

Source: authors

The claims reported each year are characterized approximately by the following delay in their payments: $50 \%$ in the actual year, $35 \%$ in the next year and $15 \%$ in the year after next. Table 8 contains also further adjustments of the amount necessary to cover the insurance claims reported to 31 December 2015 due to the inflation (e.g. $4063889 \cdot 1.05^{2}=4480438$ ) and due to the time value of money adjusted by discounting (e.g. $\left.4480438 / 1.04^{2}=4142416\right)$.

Then one calculates easily the mean claim of the type IBNR in the year 2015

$$
A_{2015}=11796186 / R_{2015}=11796186 / 10500=1123 \text { EUR } \text {. }
$$

Since it was found empirically in the considered situation that starting with $N_{2012}=100$ claims of the type IBNR in the year 2012 one has reported 85 claims in the year 2013 and 10 claims in the year 2014, one can put

$$
p_{1}=0.85, \quad p_{2}=0.95 \text {. }
$$

Therefore after substituting to (29) one obtains

$$
N_{2015}=129 \text {. }
$$

Finally, according to (28) one obtains the best estimate of the IBNR reserve in the year 2015 as

$$
T P_{I B N R, 2015}=129 \cdot 1123=144500 \mathrm{EUR} \text {. }
$$

\section{Counterparty Default Risk}

As to the counterparty default risk, we suggest a method that can be suitable when the reinsurers form a small group of heterogeneous subjects impacted by a common shock as a financial crisis or a natural catastrophe (so far only "asymptotic" methods for large portfolios of homogenous risks are usual in literature, see e.g. CEA, 2006; McNeil et al., 2005; Sandström; 2011). 


\subsection{Description of method based on common shock}

The suggested method can be described in several steps:

(i) Let us denote the common shock affecting all reinsurers of a given insurance company (e.g. the arrival of the financial and economic crisis or recession, a legislative change or reform, a catastrophic event) as a random variable $R$ ranging in the interval between zero and one. For the values of $R$ near to zero or one, the common influence of the given phenomenon is low or high, respectively. The behaviour of $R$ can be described by the probability density function of the form

$$
f(r)=\beta r^{\beta-1}, \quad 0<r<1,
$$

where $\beta$ is a parameter $(0<\beta<1)$. Such a probability density function can be looked upon as a special case of the beta distribution (e.g. Cipra, 2015), and it is acceptable from the practical point of view since according to it (1) the small shocks are the most probable ones, while (2) the probability of more intensive shocks declines to small positive values (however, the zero value is never achieved). Another interpretation is also possible: the maximal shock among $n$ mutually independent annual shocks (i.e. during $n$ years) has the probability density function of the form (30) but with the parameter $n \beta$ instead of $\beta$. Therefore e.g. for $\beta=0.05$ and $n=20$, the probability density function (30) corresponds to the uniform distribution so that the maximal shock during the period of 20 years attains each of its values with the same probability (one can make use of this property when calibrating the parameter $\beta$ ).

(ii) The probabilities of default will depend on the common shock $R$. A suitable functional relation seems to be

$$
P D_{i}(r)=p_{i}+\left(1-p_{i}\right) r^{\gamma / p_{i}}, \quad 0<r<1,
$$

where $p_{i}$ is a basic level of the probability of default of the $i$-th counterparty $(i=1, \ldots, k)$ in the credit portfolio (it is a benchmark if excluding the influence of the common shock $R$ ). Moreover, in (31) one adds to this basic level a component that depends on $R$ by means of a positive parameter $\gamma$. The power exponent in (31) is a decreasing function of the basic level of the probability of default $p_{i}$ since counterparties with a low $p_{i}$ are not very sensitive to the random shocks while the higher probability of default increases the default sensitivity even if the attained values of $R$ are not high. In any case, the function (31) increases from the basic level $p_{i}$ to the value 1. Moreover, this function is concave for $\gamma<p_{i}$ (and the probability of default $P D_{i}$ is considered more likely as large), it is convex for $\gamma>p_{i}$ (and the probability $P D_{i}$ is considered more likely as small); for $\gamma=p_{i}$ one obtains an increasing line.

(iii) By integrating the function (31) over $r$ using the probability density function (30) one obtains the formula for the probability of default of the $i$-th counterparty as

$$
P D_{i}=\mathrm{E}\left(P D_{i}(R)\right)=\int_{0}^{1} P D_{i}(r) f(r) d r=\frac{(\gamma+\beta) p_{i}}{\gamma+\beta p_{i}} .
$$


Conversely, if one takes the numerical values of default probabilities $P D_{i}$ of particular counterparties as an external rating provided by specialized agencies, e.g. Standard \& Poor's (S\&P) or other subjects which rate regularly reinsurance companies, then one can find the basic level of the probability of default $p_{i}$ of the $i$-th counterparty evidently as

$$
p_{i}=\frac{\gamma P D_{i}}{\beta\left(1-P D_{i}\right)+\gamma} .
$$

One can summarize that the behaviour of particular counterparties in the credit portfolio may be modelled in a suitable parametric way using two parameters $\beta$ and $\gamma$.

(iv) Finally, one should extend the results for particular counterparties to the whole credit portfolio. Let $I_{i}$ is a random default indicator of the $i$-th counterparty, i.e. $I_{i}=1$ or $I_{i}=0$ depending whether the default has occurred or not, respectively. The loss $L$ generated by the whole credit portfolio can be expressed in the form

$$
L=\sum_{i=1}^{k} L G D_{i} \cdot I_{i}
$$

where $L G D_{i}$ is the particular loss following from the default of the $i$-th counterparty. It holds

$$
\mathrm{E}(L)=\sum_{i=1}^{k} L G D_{i} \cdot P D_{i}, \quad \operatorname{var}(L)=\sum_{i=1}^{k} \sum_{j=1}^{k} L G D_{i} \cdot L G D_{j} \cdot \sigma_{i j},
$$

where

$$
\sigma_{i i}=P D_{i}\left(1-P D_{i}\right), \quad \sigma_{i j}=\frac{\beta\left(1-p_{i}\right) \cdot\left(1-p_{j}\right)}{\beta+\gamma p_{i}^{-1}+\gamma p_{j}^{-1}}-\left(P D_{i}-p_{i}\right) \cdot\left(P D_{j}-p_{j}\right), \quad i \neq j .
$$

For instance, to derive $\sigma_{i j}$ for $i \neq j$ one can write using (32)

$$
\begin{gathered}
\sigma_{i j}=\operatorname{cov}\left(I_{i}, I_{j}\right)=E\left(I_{i}, I_{j}\right)-E\left(I_{i}\right) \cdot E\left(I_{j}\right)=E\left(I_{i}, I_{j}\right)-P D_{i} \cdot P D_{j}= \\
=\int_{0}^{1}\left[p_{i}+\left(1-p_{i}\right) r^{\gamma / p_{i}}\right]\left[p_{j}+\left(1-p_{j}\right) r^{\gamma / p_{j}}\right] \beta r^{\beta-1} \mathrm{~d} s-\frac{(\gamma+\beta) p_{i}}{\gamma+\beta p_{i}} \cdot \frac{(\gamma+\beta) p_{j}}{\gamma+\beta p_{j}}= \\
=\frac{\beta\left(1-p_{i}\right) \cdot\left(1-p_{j}\right)}{\beta+\gamma p_{i}^{-1}+\gamma p_{j}^{-1}}-\left(P D_{i}-p_{i}\right) \cdot\left(P D_{j}-p_{j}\right) .
\end{gathered}
$$

In the special case of a single counterparty (i.e. $k=1$ ) the relations (35) and (36) are simplified to

$$
\mathrm{E}(L)=L G D \cdot P D, \quad \operatorname{var}(L)=L G D^{2} \cdot P D \cdot(1-P D)
$$

Hence the capital requirements calculated using the quantile risk measures can be obtained (e.g. value-at-risk VaR and others, see Cipra, 2015). 


\subsection{Numerical demonstration}

Table 9 contains numerical values that are used for the calculation of the credit capital requirements as a numerical example of the methodology described in Section 5.1. In this example an insurer is reinsured by three reinsurers $(i=1,2,3)$ with the default probabilities corresponding to their external rating according to $\mathrm{S} \& \mathrm{P}$ (the applied values of parameters $\beta$ and $\gamma$ are taken from Cipra (2015), but in practice they should be prescribed by the regulator).

Table 9 | Numerical Values Used for the Calculation in Section $\mathbf{5 . 2}$

\begin{tabular}{|l|c|c|}
\hline \multirow{3}{*}{ Parameters } & Symbol & Numerical value \\
\hline \multirow{3}{*}{$\begin{array}{l}\text { Probability of default } \\
\text { (S\&P) }\end{array}$} & $\beta$ & 0.05 \\
\cline { 2 - 3 } & $\gamma$ & 0.1 \\
\cline { 2 - 3 } & $P D_{1}(\sim A A)$ & 0.005 \\
\hline \multirow{3}{*}{ Loss given default } & $P D_{2}(\sim A)$ & 0.008 \\
\cline { 2 - 3 } & $P D_{3}(\sim B B B)$ & 0.083 \\
\cline { 2 - 3 } & $L G D_{1}$ & $300 \mathrm{~m} \mathrm{CZK}$ \\
\hline
\end{tabular}

Source: authors

In Table 10 we have calculated the basic levels of the probabilities of default of particular counterparties (according to (33)) and the variances $\sigma_{i i}$ and covariances $\sigma_{i j}$ (according to (36)) necessary for the calculation of variance of loss in (35).

Table 10 | Auxiliary Values Calculated in Section $\mathbf{5 . 2}$

\begin{tabular}{|l|c|c|c|c|}
\hline \multirow{2}{*}{$\boldsymbol{i}$} & \multirow{2}{*}{$\boldsymbol{p}_{\boldsymbol{i}}$} & \multicolumn{3}{|c|}{$\boldsymbol{\sigma}_{\boldsymbol{i j}}$} \\
\cline { 3 - 5 } & & $\boldsymbol{j}: \mathbf{1}$ & $\mathbf{2}$ & $\mathbf{3}$ \\
\hline $\mathbf{1}$ & 0.003 & 0.005 & 0.001 & 0.001 \\
\hline $\mathbf{2}$ & 0.005 & 0.001 & 0.008 & 0.002 \\
\hline $\mathbf{3}$ & 0.057 & 0.001 & 0.002 & 0.076 \\
\hline
\end{tabular}

Source: authors

It enables to calculate according to (35) the expected value and the standard deviation of the loss $L$ following from the credit default of counterparties 


$$
\mathrm{E}(L)=9.3 \mathrm{~m} \mathrm{CZK}, \sqrt{\operatorname{var}(L)}=36.4 \mathrm{~m} \mathrm{CZK} .
$$

Assuming the normal distribution one obtains finally e.g. $\operatorname{VaR}_{0.995}=103.2 \mathrm{~m} \mathrm{CZK}$ (i.e. the value-at-risk for the confidence level 99.5\%).

\section{Conclusions}

The paper concentrated on important aspects of the underwriting risk and counterparty credit risk in the framework of Solvency II.

In particular, the problem of technical provisions was investigated carefully since the results of the quantitative impact studies QIS (e.g. Cipra, 2015) have shown that the technical provisions constructed according to this new methodology (e.g. (14)) may be substantially lower (sometimes even by $15 \%$ ) than the ones constructed according to the practice before Solvency II. The simplified calculation of technical provisions shown in the paper can be useful in the actuarial practice.

A specific approach to the counterparty credit risk based on the concept of common shock was also suggested. This approach should be extended in a future research in order to enable not only the calculation of $V a R$ without the assumption of normality but also the calculation of other quantile risk measures (e.g. the conditional value-at-risk $C V a R$, expected shortfall $E S$, marginal expected shortfall MES) since the VaR recommended in the so called standard formula of Solvency II is overcome in the new risk regulatory scheme Basel III for banks (see BIS, 2010).

\section{References}

BIS (2010). A Global Regulatory Framework for More Resilient Banks and Banking Systems. Basel: Bank for International Settlements. ISBN 92-9131-859-0.

CEA (2006). CEA Working Document on the Standard Approach for Calculating the Solvency Capital Requirement. Brussels: Comite Europeen des Assurances.

Cipra, T. (2010). Financial and Insurance Formulas. Heidelberg, Dordrecht, London, New York: Physica-Verlag/Springer. ISBN 978-3-7908-2592-3, https://doi. org/10.1007/978-3-7908-2593-0

Cipra, T. (2015). Risk in Finance and Insurance: Basel III and Solvency II. Prague: Ekopress (in Czech).

GDV (2015), Discussion Paperfor a Solvency ll Compatible Standard Approach (Pillarl) Model Description. Berlin: Gesamtverband der Deutschen Versicherungswirtschaft.

Hendrych, R., Cipra, T. (2015). Econometric Model of the Czech Life Insurance Market. Prague Economic Papers, 24(2), 173-191, https://doi.org/10.18267/j.pep.507

Hürlimann, W. (2008). On the Non-life Solvency II Model. Manchester: 38th ASTIN Colloquium.

IAA (2004). A Global Framework for Insurance Solvency Assessment. Ontario: International Actuarial Association.

Mack, T. (1993). Distribution-Free Calculation of the Standard Error of Chain Ladder Reserves Estimates. ASTIN Bulletin, 23(2), 213-225, https://doi.org/10.2143/ast.23.2.2005092

McNeil, A. J., Frey, R., Embrechts, P. (2005). Quantitative Risk Management. Princeton and Oxford: Princeton University Press. 
Merz, M., Wüthrich, M. V. (2008). Modeling the Claims Development Result for Solvency Purposes. Manchester: 38th ASTIN Colloquium.

Ohlsson, E., Lauzeningks, J. (2008). The One-Year Non-life Insurance Risk. Manchester: 38th ASTIN Colloquium.

Sandström, A. (2011). Handbook of Solvency for Actuaries and Risk Managers. Theory and Practice. New York: Chapman and Hall/CRC Press.

SST (2006). Technical Document on the Swiss Solvency Test. Switzerland: Federal Office of Private Insurance.

Wüthrich, M. V., Merz, M. (2008). Stochastic Claims Reserving Methods in Insurance. Chichester: Wiley. 